\title{
Numerical investigation of conjugate heat transfer and flow performance of a fin and tube heat exchanger with vortex generators
}

\author{
Shobhana Singh Kim Sørensen \\ Department of Energy Technology, Aalborg University, Pontoppidanstræde 111, 9022, Aalborg, Denmark, \\ \{ssi,kso\}@et.aau.dk
}

\begin{abstract}
Vortex generator is considered as an effective device for augmentation of the thermal-hydraulic performance of a heat exchanger. The aim of present study is to examine the influence of vortex generators on a double fin and tube heat exchanger performance. Vortex generator of rectangular winglet type is chosen and investigated at different angles of attack $0^{\circ},-10^{\circ}$ and $-20^{\circ}$ with the flow direction. Three-dimensional numerical model is developed and simulations are performed for a Reynolds number range $5000 \leq R e \leq 11000$ taking conjugate heat transfer into account. The heat transfer and pressure loss characteristics are determined and analyzed for an in-line configuration of a fin and tube heat exchanger. In order to evaluate the enhancement in the performance on an equitable basis, the heat exchanger with plain fin surface is considered as a reference design. Results show that the angle of attack of a vortex generator has a significant impact on the volume goodness factor, and enhance the thermal performance of a fin and tube heat exchanger in comparison to the design with plain fin. The vortex generator at an angle of attack $-10^{\circ}$ is found to perform superior over the Reynolds number range studied. At $R e=5000$, the vortex generator with an angle of attack $-10^{\circ}$ increases the volume goodness factor by $32 \%$ with respect to the baseline heat exchanger design.
\end{abstract}

Keywords: Fin and tube heat exchanger, vortex generator, conjugate heat transfer, volume goodness factor

\section{Introduction}

Fin and tube heat exchangers are one of the most commonly used thermal engineering systems due to the larger heat transfer area provided by the extended surface integrated tubes. In the process industry, vortex generators (VGs) are being increasingly incorporated in modern multi-functional heat exchangers to enhance heat and mass transfer and to increase energy efficiency (Ghanem et al. , 2013). The enhancement by vortex generators shows a great promise in reducing the size of heat exchangers (Biswas et al. , 1994). They are integrated into a surface by means of embossing, stamping, punching, or by other attachment processes.
Wide range of application and design flexibility have made vortex generators an effective mode of performance enhancement. In fin and tube heat exchanger often negligible heat transfer takes place in the downstream of the circular tube due to a low velocity fluid which can be enhanced by several hundred percent by placing VG in the wake region behind the tubes (Biswas et al. , 1994; Joardar and Jacobi , 2008). Several experimental and numerical studies on different configurations of VGs have been carried out over the last two decades (Turk and Junkhan , 1986; Joardar and Jacobi , 2008; Tiggelbeck et al. , 1992, 1993; Jacobi and Shah , 1995; Jang et al. , 2013) including fin and tube heat exchangers (Fiebig, 1995; Fiebig et al. , 1994, 1993). However, most of the studies are commonly based on convective heat transfer analysis.

In majority of engineering applications, for example, waste heat recovery, heating and cooling, aerospace engineering etc., physical phenomena usually combines both heat transfer in solid and fluids. For accurate predictions of improved performance for a given application, numerical simulations with coupled temperature fields in solids and fluids are essential. It is pragmatic in testing different design configurations with vortex generators. Due to the lack of sufficient data on the impact of VGs on doubled fin and tube heat exchangers, present study work aims to investigate the effect of VGs with different angles of attack $(\alpha)$ on the heat transfer and pressure loss characteristics. The analysis is performed by developing a three-dimensional (3D) numerical model that includes conjugate heat transfer in solid and fluid domains simultaneously.

\section{Numerical Model}

\subsection{Computational Geometry: Fin and Tube Heat Exchanger with Vortex Generators}

Figure 1a shows a double fin and tube heat exchanger with plain fin and with VGs as investigated in the present study. The double fin and tube heat exchanger is generally designed for a wide range of applications such as marine exhaust gas boilers for waste heat recovery from coal-fired boilers (Singh et al., 2016a,b, 2017) due 


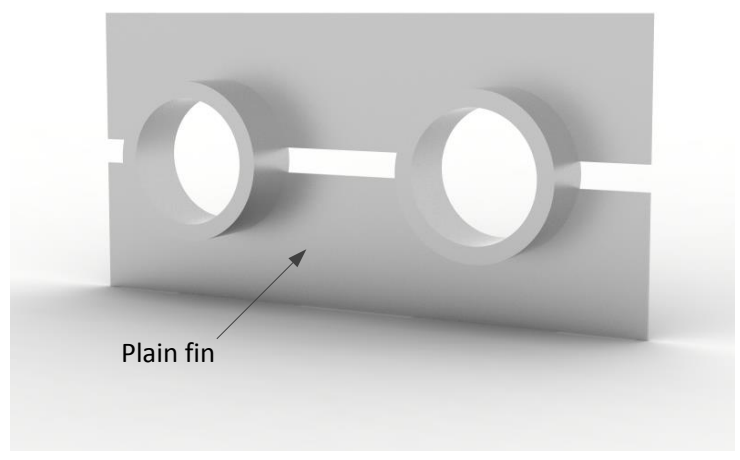

(a) Plain fin

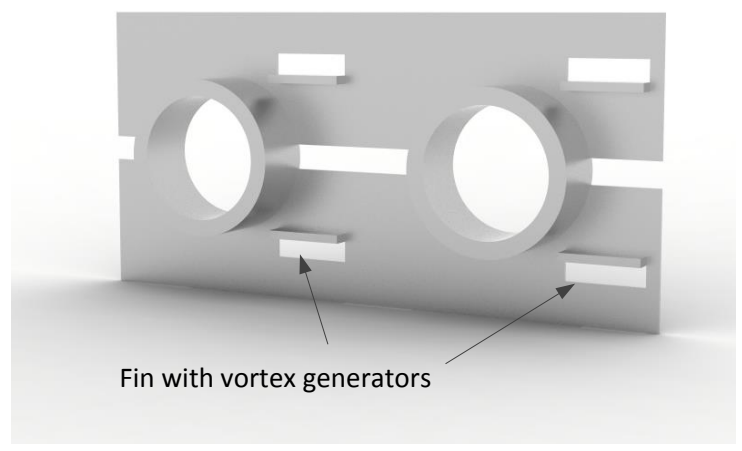

(b) Fin with vortex generator

Figure 1. Illustration of fin and tube heat exchanger design

to good anti-wear and anti-fouling performance, compact design, stability (Chen and Lai, 2012; Jin et al., 2013, 2016). To investigate the effectiveness of VGs, rectangular winglet vortex generator (RWVG) are selected considering their heat transfer enhancement performance better than delta winglets (Li et al., 2015). Two pairs of RWVGs each of height $0.004 \mathrm{~m}$ and length $0.019 \mathrm{~m}$ are placed longitudinally on the fin surface in the direction of the flow. The rectangular winglets can be easily manufactured by punching out off the fin surface at an angle of $90^{\circ}$ as shown in Fig.1b. Figure 2 illustrates the design details of the rectangular vortex generator at an angles of attack $\alpha$ with the incoming flow direction. Table 1 lists the design parameters of the heat exchanger considered in the study.

\subsection{Governing Equations}

Based on flow velocity and Reynolds number ( $\geq 5000$ ), Shear Stress Transport (SST) turbulence model is adopted to simulate the fluid flow in the domains of interest (Menter, 1994; Menter et al., 2003; Woelke, 2007).

The governing equations of mass, momentum, and
Table 1. Dimensions of the computational geometry simulated in the present study

\begin{tabular}{ll}
\hline Parameter & Value \\
\hline Length of the fin, $L_{f}$ & $0.145 \mathrm{~m}$ \\
Width of the fin, $W_{f}$ & $0.032 \mathrm{~m}$ \\
Thickness of the fin base, $\delta_{f}$ & $0.002 \mathrm{~m}$ \\
Width of the gap between fins, $d_{f}$ & $0.006 \mathrm{~m}$ \\
Fin pitch, $p_{f}$ & $0.015 \mathrm{~m}$ \\
Inner diameter of the tube, $D_{i}$ & $0.030 \mathrm{~m}$ \\
Outer diameter of the tube, $D_{o}$ & $0.038 \mathrm{~m}$ \\
Tube pitch, $p_{t}$ & $0.075 \mathrm{~m}$ \\
Length of the gas domain, $L_{g}$ & $0.150 \mathrm{~m}$ \\
Width of the gas domain, $W_{g}$ & $0.075 \mathrm{~m}$ \\
Thickness of the gas domain, $\frac{p_{f}}{2}$ & $0.0075 \mathrm{~m}$ \\
Length of RWVG, $l_{V G}$ & $0.019 \mathrm{~m}$ \\
Height of RWVG, $h_{V G}$ & $0.004 \mathrm{~m}$ \\
Thickness of RWVG, $\delta_{V G}$ & $0.002 \mathrm{~m}$ \\
Angle of attack of RWVG, $\alpha$ & $0^{\circ},-10^{\circ},-20^{\circ}$ \\
\hline
\end{tabular}

energy conservation can be written as:

$$
\begin{gathered}
\frac{\partial \rho}{\partial t}+\nabla(\rho \mathbf{U})=0 \\
\frac{\partial(\rho \mathbf{U})}{\partial t}+\nabla(\rho \mathbf{U} \otimes \mathbf{U})-\nabla\left(\mu_{e f f}-\nabla \mathbf{U}\right)=-\nabla p \\
+\nabla\left(\mu_{e f f}-\nabla \mathbf{U}\right)^{T}+\mathbf{F}
\end{gathered}
$$

where $\mathbf{U}$ is the averaged flow velocity field $[\mathrm{m} / \mathrm{s}], \rho$ is the density $\left[\mathrm{kg} / \mathrm{m}^{3}\right], \otimes$ is the outer vector product, $p$ is the pressure $[\mathrm{Pa}], \mathrm{F}$ is the total body force $\left[\mathrm{N} / \mathrm{m}^{3}\right], \mu_{e f f}$ is the effective viscosity coefficient $[P a . s]$.

$$
\frac{\partial\left(\rho C_{p} T\right)}{\partial t}+\nabla\left(\rho C_{p} \mathbf{U}\right) T=-\nabla(\mathbf{q})+\tau: \nabla \mathbf{U}-p \nabla \mathbf{U}+\mathbf{Q}
$$

$$
\frac{\partial\left(\rho C_{p} T\right)}{\partial t}=-\nabla(\mathbf{q})+\mathbf{Q}
$$

where $C_{p}$ is the specific heat capacity at constant pressure $[\mathrm{J} / \mathrm{kgK}], \mathbf{q}$ is the heat flux by conduction $\left[\mathrm{W} / \mathrm{m}^{2}\right], \vec{\tau}: \nabla \vec{U}$ is the viscous heating term and is assumed negligible, $\mathbf{Q}$ is the heat source $\left[\mathrm{W} / \mathrm{m}^{3}\right]$.

The transport equations of SST model in terms of $k$ and $\omega$ can be written as:

$$
\begin{gathered}
\left.\frac{\partial(\rho \omega)}{\partial t}+\nabla(\rho \omega \mathbf{U})=\nabla\left[\left(\mu+\frac{\mu_{t}}{\sigma_{\omega, 1}}\right) \nabla(\omega)\right)\right]+ \\
\gamma_{2}\left[2 \rho S_{i j} S_{i j}-\frac{2}{3} \rho \omega \frac{\partial U_{i}}{\partial x_{j}} \delta_{i j}\right]-\beta_{2} \rho \omega^{2}+2 \frac{\rho}{\sigma_{\omega, 2} \omega} \frac{\partial k}{\partial x_{k}} \frac{\partial \omega}{\partial x_{k}}
\end{gathered}
$$

where $\omega$ is the turbulent frequency $[1 / s]$ and $\mu_{t}=\frac{\rho k}{\omega}$ is the eddy viscosity. 

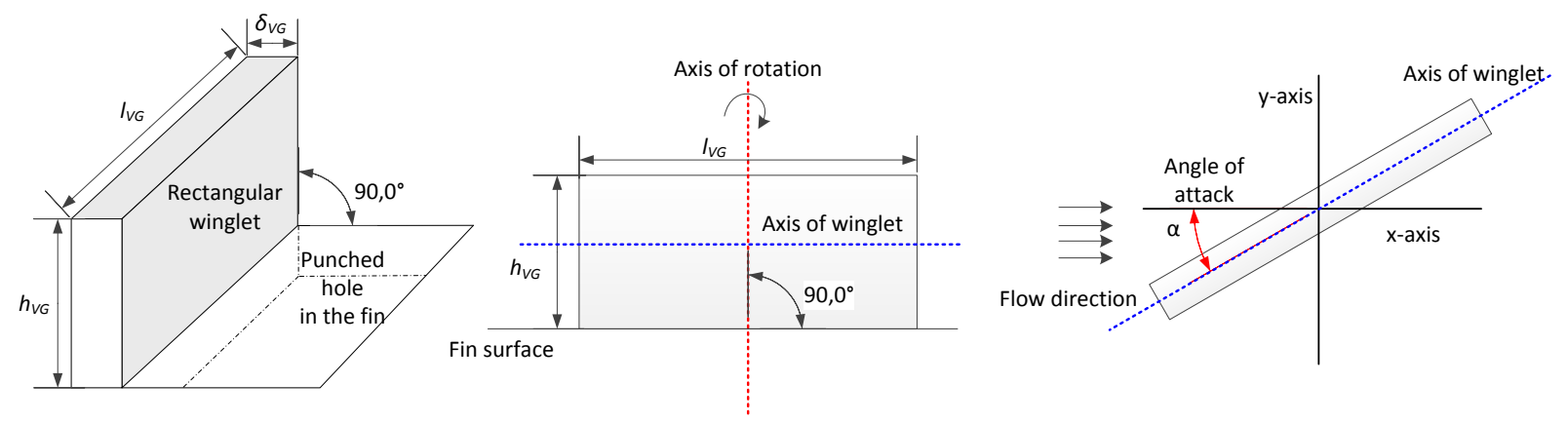

Figure 2. Schematic illustration of rectangular winglet vortex generator

$$
\left.\frac{\partial(\rho k)}{\partial t}+\nabla(\rho k \mathbf{U})=\nabla\left[\left(\mu+\frac{\mu_{t}}{\sigma_{k}}\right) \nabla(k)\right)\right]+P_{k}
$$

where $k$ is the rate of turbulent kinetic energy $\left[\mathrm{m}^{2} / \mathrm{s}^{2}\right]$.

The rate of production of turbulent kinetic energy which is given as:

$$
P_{k}=\left(2 \mu_{t} S_{i j} . S_{i j}-\frac{2}{3} \rho k \frac{\partial U_{i}}{\partial x_{j}} \delta_{i j}\right)
$$

\subsection{Solution Procedure}

In most of the numerical problems, Computational Fluid Dynamics (CFD) based tool solves the governing transport equations faster under specified conditions and predicts the physical processes such as fluid flow, heat transfer etc. with reliable accuracy. In the present work, Ansys CFX v.17.1.(Ansys , 2017) is used to develop the finite volume model and simulate steady state flow with coupled conjugate heat transfer. Control surface approach is used to perform the coupling between the fluid and solid grid interface. To gain the time savings and reduced computational power, symmetric boundary conditions are employed as shown in the Fig.3. The computational geometry has faces set as a plane of symmetry and periodic inlet and outlet. The boundary conditions at different faces of the computational domain are described in Table 2. Due to a high complexity design including VGs, unstructured mesh is generated with a fine boundary layers. Meshes with $\approx 7000000$ and $\approx 13000000$ average number of grid elements are selected for plain fin and fin with VG to achieve the reliable numerical solution, respectively. The convergence is obtained when the maximum residual of objective variables (i.e. temperature, pressure and velocity components) accomplished $10^{-4}$.

\subsection{Model Validation}

In order to validate the model, the Nusselt number and Euler number values from the correlations of Chen et al.
Table 2. Boundary conditions used to solve the numerical model

\begin{tabular}{ll}
\hline Boundary & Conditions \\
\hline Periodic & $m=m_{i n}$ \\
Wall & $\mathbf{U} \cdot \mathbf{n}=0 ; \quad k=0 ;$ \\
& {$\left[\lim _{l_{\omega} \rightarrow 0} \omega=\frac{6 \mu}{\rho \beta l_{w^{2}}}\right]$} \\
Symmetry & $\mathbf{U} \cdot \mathbf{n}=0 ; \nabla \cdot k=0 ; \nabla . \omega$ \\
& $=0$ \\
Inner tube wall & $T=T_{w}$ \\
\hline Operating & Conditions \\
\hline Gas temperature, $T_{i n}$ & $573.15 K$ \\
Inner tube wall temperature, $T_{w}$ & $453.15 K$ \\
\hline
\end{tabular}

(2014) are compared with the present numerical results as shown in Fig.4. The standard deviation of predicted values of Nusselt and Euler numbers from the values obtained from the correlations values vary from 0.004 to 5.150 , respectively. The deviation can be justified by the assumptions made in model parameters in addition to the experimental error in developing the correlations. The lower standard deviation in the numerical values indicates the reliability and validity of the computational model under given operating conditions.

\section{Data Analysis and Performance Evaluation}

In this section, the heat transfer and flow characteristics are defined to evaluate the performance enhancement in the fin and tube heat exchanger by RWVGs.

Reynolds number in the present study is determined as:

$$
R e=\frac{u_{i n} D_{o}}{v}
$$

where $R e$ is the Reynolds number [-], $u_{\text {in }}$ is the inlet flow velocity $[\mathrm{m} / \mathrm{s}], D_{o}$ is the outer tube diameter $[\mathrm{m}]$ and $v$ is the kinematic viscosity of the air $[\mathrm{m} / \mathrm{s}]$.

The overall avergae heat transfer coefficient of the fin and tube heat exchanger is calculated as:

$$
\bar{h}=\frac{Q}{A_{t} \Delta T_{l m}}
$$




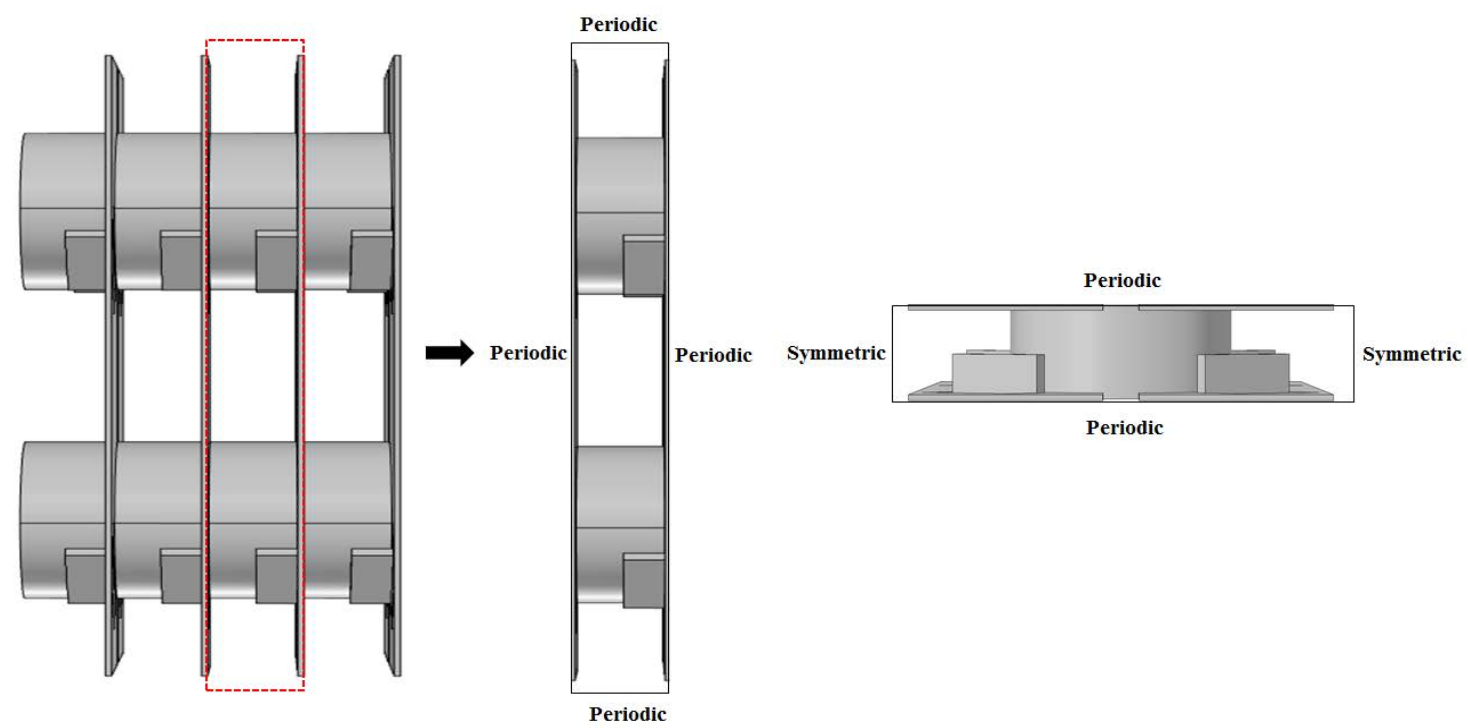

Figure 3. Computational geometry simulated in the present study

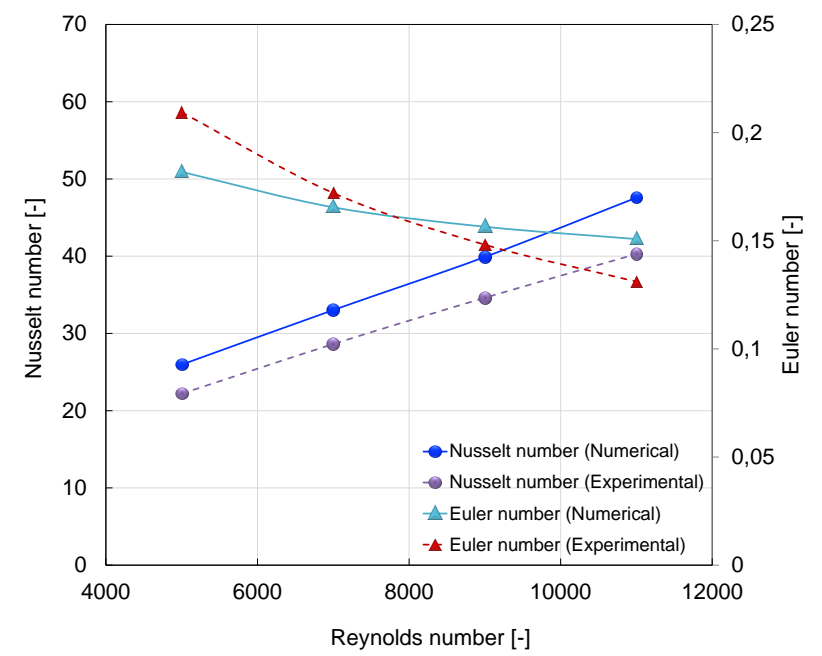

Figure 4. Numerical model validation based Nusselt number and Euler number correlations

where $\bar{h}$ is the overall heat transfer coefficient $\left[\mathrm{W} / \mathrm{m}^{2}\right.$. $K], A_{t}$ is the total heat transfer area $\left[\mathrm{m}^{2}\right]$, and $Q$ is the total heat transferred calculated as:

$$
Q=\dot{m} C_{p}\left(T_{\text {in }}-T_{\text {out }}\right)
$$

where $\dot{m}$ is the mass flow rate $[\mathrm{kg} / \mathrm{s}], C_{p}$ is the specific heat capacity $[\mathrm{J} / \mathrm{kg} \cdot \mathrm{K}], T_{\text {in }}$ and $T_{\text {out }}$ are the mass averaged air temperature at the inlet and outlet $[K] . \Delta T_{l m}$ is the logarithmic mean temperature difference which is calculated as:

$$
\Delta T_{l m}=\frac{\left(T_{\text {in }}-T_{w}\right)-\left(T_{\text {out }}-T_{w}\right)}{\ln \left(\frac{T_{\text {in }}-T_{w}}{T_{\text {out }}-T_{w}}\right)}
$$

where $T_{w}$ is the inner tube wall temperature $[K]$.

In order to determine heat transfer characteristic, average Nusselt number which is calculated as:

$$
\overline{N u}=\frac{\bar{h} D_{o}}{k}
$$

Colburn- $j$ factor is calculated based on average Nusselt number and is expressed as:

$$
j=\frac{\overline{N u}}{\operatorname{RePr}^{1 / 3}}
$$

where $\rho$ is the density of the fluid $\left[\mathrm{kg} / \mathrm{m}^{3}\right], P r$ is the Prandtl number [-], and $\overline{N u}$ is the

In addition to the heat transfer characteristics, pressure losses are be evaluated using the Euler number, $E u$ and the friction factor, $f$ as:

$$
\begin{gathered}
E u=\frac{\Delta p}{\frac{1}{2} \rho u_{\max }^{2}} \\
f=\frac{\Delta p}{\frac{1}{2} \rho u_{\text {in }}^{2}} \times \frac{D_{o}}{L_{g}}
\end{gathered}
$$

where, $\Delta p$ is the pressure loss $[\mathrm{Pa}]$ and $L_{g}$ is the length of the gas domain in the computational geometry $[\mathrm{m}]$

Due to a number of possible enhancement methods, operating conditions and available designs, comparison of performance on equitable basis can be challenging. In the present study, a factor called Volume goodness factor as proposed by Kays and London (1998) is utilized to predict the overall performance. It considers simultaneous effect of heat transfer and pressure loss and, therefore, useful in several practical applications.

$$
\frac{j}{f^{1 / 3}}=\frac{\frac{\overline{N u}}{\operatorname{RePr}^{1 / 3}}}{\left(\frac{\Delta p}{\frac{1}{2} \rho u_{\text {in }}^{2}} \times \frac{D_{o}}{L_{g}}\right)^{1 / 3}}
$$




\section{Results and Discussion}

\subsection{Heat Transfer Enhancement}

The influence of angle of attack $(\alpha)$ on Nusselt number can be seen in Fig.5a. It is clear that heat exchanger with RWVG has higher Nusselt number than that of baseline plain fin surface which increases with a increase in Reynolds number at a given $\alpha$. VGs induce stronger vortical fluid flow due to which heat transfer coefficient becomes higher, thereby, augments the heat transfer between the fluid and surface. It is observed that Nusselt number increases as $\alpha$ varies from $0^{\circ}$ to $-20^{\circ}$, however, the increment becomes insignificant as $\alpha$ goes higher than $0^{\circ}$.

To make a rational comparison between the heat transfer performance at different $\alpha$, Colburn- $j$ factor normalized with the baseline plain fin value is plotted in Fig.5b. It can be seen that adding RWVG can enhance the heat transfer by up to 55\% at a given Reynolds number. Moreover, it is interesting to note that Colburn- $j$ factor ratio of fins with RWVG at $\alpha=-10^{\circ}$ and $\alpha=-20^{\circ}$ decreases as the Reynolds number increases while remains more or less constant at $\alpha=0^{\circ}$ over the Reynolds number range. The results indicate that RWVGs in double fin and tube heat exchanger tend to loose their advantage relative to baseline plain fin at higher Reynolds number.

\subsection{Induced Flow Loss}

Enhancement in heat transfer in most of the applications is accompanied with a penalty of pressure loss. To account for this penalty, the Euler number with a different angle of attack is plotted against Reynolds number in Fig. 6a. The figure shows that Euler number increases as $\alpha$ vary from $0^{\circ}$ to $-20^{\circ}$. At $\alpha=-20^{\circ}$, Euler number is the highest which is due to the increased fluid velocity and consequently large pressure loss. The RWVG with $\alpha=0^{\circ}$ performs nearly similar to the baseline plain fin design.

Another factor called friction factor $(f)$ is evaluated using Eq.15. Figure 6b compares the normalized friction factor at different angles of attack to the baseline plain fin design. It can be seen that friction factor ratio remains nearly constant over the entire range of Reynolds number, however, fins with RWVGs have higher friction factor than baseline plain fin and the highest at $\alpha=-20^{\circ}$. At $\alpha=-20^{\circ}$ and $R e=11000$, friction factor is $20 \%$ higher than that of baseline plain fin design. It is demonstrated by the results that adding RWVG to the fin surface increases the pressure loss which certainly is a disadvantage to the overall performance.

It is clear from the results that the angle of attack of RWVG has a significant impact on thermal and pressure loss characteristics of a fin and tube heat exchanger. It is, therefore, essential to analyze both characteristics together. In the present study, the overall performance is evaluated using Volume goodness factor from Eq.16.

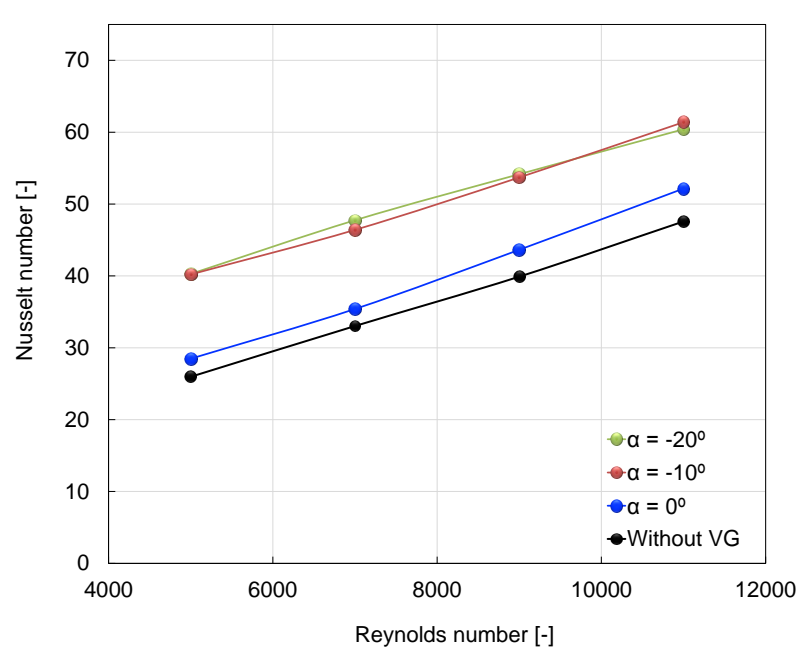

(a) Nusselt number

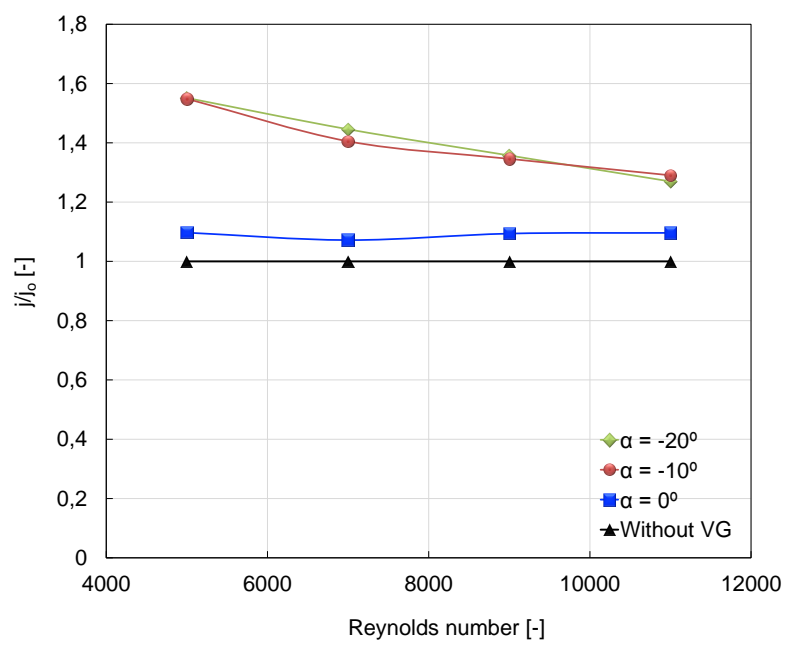

(b) Colburn $\mathrm{j}$ factor

Figure 5. Heat transfer characteristics with RWVG at different angle of attack

Figure 7 compares the factor with the plain fin factor as a reference. it is found that RWVG at $\alpha=-10^{\circ}$ has higher volume goodness factor followed by $\alpha=-20^{\circ}$ over the entire range of Reynolds number in this study which indicates that $\alpha=-10^{\circ}$ performs better in comparison to RWVG at other angles of attack when both heat transfer and pressure losses are considered together. The RWVG at $\alpha=-10^{\circ}$ and $R e=5000$ has $32 \%$ higher volume goodness factor than that of conventional plain fin surface.

It is important to emphasize that the overall performance of the heat exchanger with RWVG at $\alpha=-20^{\circ}$ and $\alpha=-10^{\circ}$ decreases as the Reynolds number increases which indicate that the advantage of 


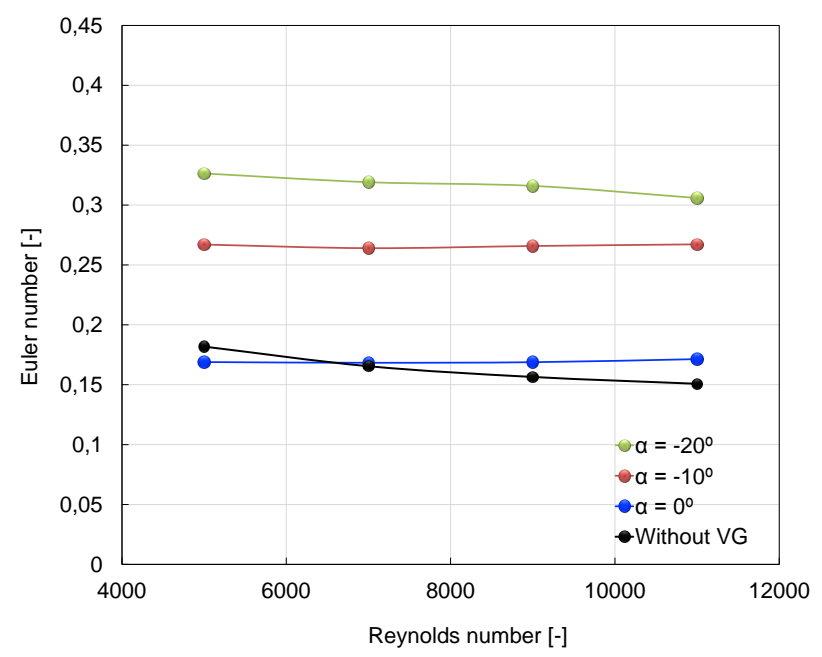

(a) Euler number

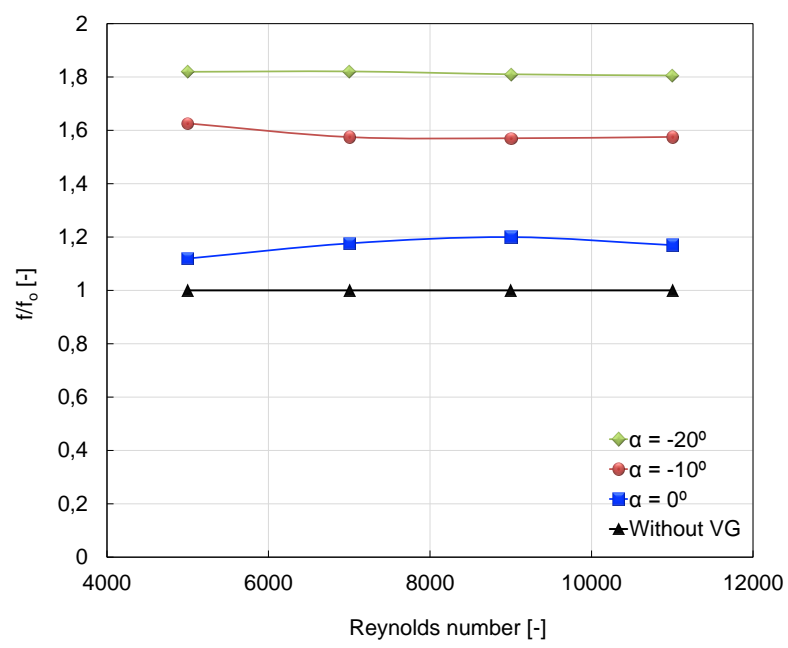

(b) Friction factor

Figure 6. Pressure loss evaluation with RWVG at different angle of attack

using RWVG at these angles of attack becomes less useful for higher Reynolds number. This is due to relatively lower heat transfer and higher pressure loss at higher Reynolds number (Figs.5b and 6b). On the other hand, performance improvement by RWVG at $\alpha=0^{\circ}$ is noticed insignificant and nearly constant at all Reynolds numbers.

A larger value of Volume goodness factor leads to reduced heat exchanger volume and weight which is desirable in most of the practical applications. Adding RWVG at $\alpha=-10^{\circ}$ in the double fin and tube heat exchanger can not only increase the overall performance but also fulfills the light weight heat exchanger demands in a number of applications.

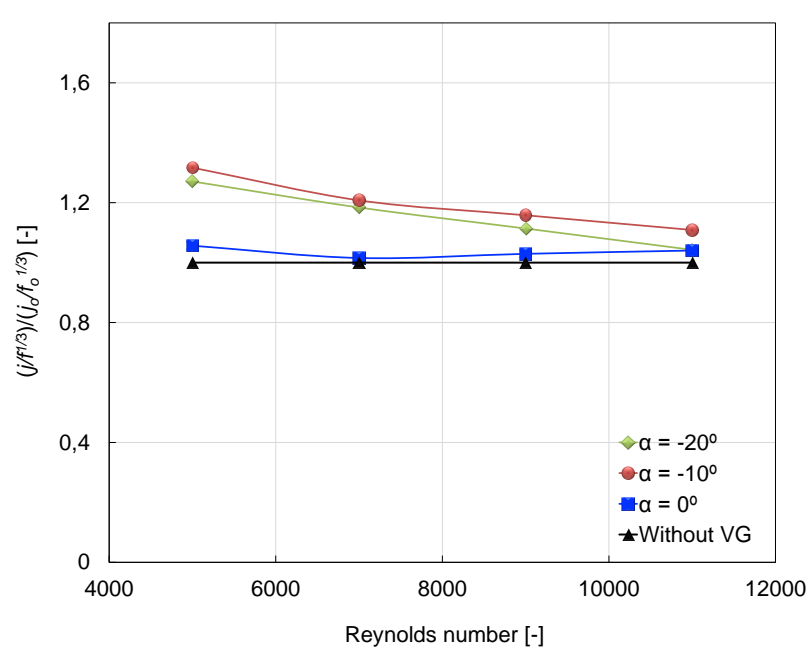

Figure 7. Overall performance comparision RWVG at different angle of attack

\section{Conclusion}

Performance enhancement using RWVGs in the double fin and tube heat exchanger is investigated numerically in the present study. The influence of different angles of attack of RWVG on heat transfer and pressure loss characteristics in relation to baseline plain fin design is demonstrated and discussed. The study can be concluded with following outcomes:

- The RWVGs significantly influence the thermal performance over the investigated range of Reynolds number i.e.5000 $\leq R e \leq 11000$. In reference to the reference design with plain fin, thermal performance is enhanced by up to $55 \%$ by RWVG with an angle of attack at $\alpha=-20^{\circ}$.

- It is interesting to note that beyond $\alpha=-10^{\circ}$, the angle of attack does not show significant improvement in thermal performance and performs similarly to $\alpha=-10^{\circ}$.

- As the angle of attack of RWVG varies from $0^{\circ}$ to $-20^{\circ}$, the pressure loss also increases. However, the trend remains more or less same over the entire range of Reynolds number studied.

- The overall performance enhancement is evaluated by comparing the volume goodness factor. Under the given operating conditions, the vortex generator at an angle of attack $-10^{\circ}$ is found to enhance the overall performance by up to $32 \%$, thus furnishes the best performed double fin and tube heat exchanger design.

- The present investigation is useful not only in enhancing the overall performance of a double fin 
and tube heat exchanger but also directs the future studies to limit the range of angle of attack of VGs while focusing on the VG location and other design parameters.

\section{References}

G. Biswas. N.K. Mitra, M. Fiebig. Heat transfer enhancement in fin-tube heat exchangers by winglet type vortex generators, International Journal of Heat and Mass Transfer, Volume 37, Issue 2, January 1994, Pages 283-291

A. Ghanem, C. Habchi, T. Lemenand, D. Della Valle, H. Peerhossaini, "Energy efficiency in process industry High-efficiency vortex (HEV) multifunctional heat exchanger", Renewable Energy, vol. 56, pp. 96-104, 2013.

A. Joardar, A.M. Jacobi, Heat transfer enhancement by winglet-type vortex generator arrays in compact plain-fin-and-tube heat exchangers, International Journal of Refrigeration, Volume 31, Issue 1, January 2008, Pages 87-97.

S. Tiggelbeck, N.K. Mitra, M. Fiebig, "Flow Structure and Heat Transfer in a Channel with Multiple Longitudinal Vortex Generators", Experimental Thermal and Fluid Science, vol. 5, pp. 425-436, 1992.

S.T. Tiggelbeck, N.K. Mitra, M. Fiebig, Experimental investigations of heat transfer enhancement and flow losses in a channel with double rows of longitudinal vortex generators, Int. J. Heat Mass Transfer 36 (1993) 2327-2337.

A.M. Jacobi, R.K. Shah, Heat transfer surface enhancement through the use of longitudinal vortices: a review of recent progress, Exp. Therm. Fluid Sci. 11 (1995) 295-309.

Jiin-Yuh Jang, Ling-Fang Hsu, Jin-Sheng Leu. Optimization of the span angle and location of vortex generators in a plate-fin and tube heat exchanger. International Journal of Heat and Mass Transfer 67 (2013) 432-444.

M. Fiebig. Embedded vortices in internal flow: heat transfer and pressure loss enhancement", International Journal of Heat and Fluid Flow 1995; 16: 376-388.

M. Fiebig, A. Valencia, N.K. Mitra. Local heat transfer and flow losses in fin-and-tube heat exchangers with vortex generators: A comparison of round and flat tubes, Experimental Thermal and Fluid Science 1994; 8(1): 35-45.

M. Fiebig, A. Valencia, N.K. Mitra. Wing-type vortex generators for fin-and-tube heat exchangers, Exp. Therm. Fluid Sci.1993; 7: 287-295.

Chen HT and Lai JR. Study of heat-transfer characteristics on the fin of two-row plate finned-tube heat exchangers.
International Journal of Heat and Mass Transfer 2012; 55: 4088-4095.

Jin Y,Tang GH, He YL and Tao WQ. Parametric study and field synergy principle analysis of H-type finned tube bank with 10 rows. International Journal of Heat Mass Transfer 2013; 60: 241-251.

Jin Y, Yu ZQ, Tang GH, He YL and Tao WQ. Parametric study and multiple correlations of an H-type finned tube bank in a fully developed region. Numerical Heat Transfer, Part A: Applications 2016; 70(1): 64-78.

A.Y. Turk, and G.H. Junkhan, "Heat Transfer Enhancement Downstream of Vortex Generators on a Flat Plate", Proceedings of the Eighth International Heat Transfer Conference, 6, pp. 2903-2908, (1986).

Chen H, Wang Y, Zhao Q, Ma H, Li Y and Chen Z. Experimental Investigation of Heat Transfer and Pressure Drop Characteristics of H-type Finned Tube Banks. Energies 2014; 7: 7094-7104.

Kays, W. M., London, A. L., Compact Heat Exchangers, Reprint 3rd ed., Krieger Publlishing Company, Malabar, Fla., USA, 1998.

Li, L.; Du, X.; Zhang, Y.; Yang, L.; yang, Y. Numerical simulation on flow and heat transfer of fin-and-tube heat exchanger with longitudinal vortex generators. Int. J. Thermal Sci. 2015, 92, 85-96.

Menter FR. Two-Equation Eddy-Viscosity Turbulence Models for Engineering Applications. AIAA Journal 1994; 38(8): 1598-1605.

Menter FR, Kuntz $M$ and Langtry R. Ten years of industrial experience with the SST Turbulence Model. Turbulence Heat and Mass Transfer 2003; 4.

Woelke M. Eddy Viscosity Turbulence Models employed by Computational Fluid Dynamic. Prace Instytutu Lotnictwa 2007; 92-113.

Singh S., Sørensen K., Condra T.J. Influence of the degree of thermal contact in fin and tube heat exchanger: A numerical analysis. Applied Thermal Engineering 2016; 107(25): 612-624.

Singh S., Sørensen K., Condra T.J. Parametric CFD Analysis to Study the Influence of Fin Geometry on the Performance of a Fin and Tube Heat Exchanger. The 9 th Eurosim Congress on Modelling and Simulation. EUROSIM 2016, 12 - 16 September 2016, Oulu Finland.

Singh S., Sørensen K., Condra, T.J. 2017. Implications of fin profiles on overall performance and weight reduction of a fin and tube heat exchanger. App. Therm. Eng. 2017; 115: 962-976.

ANSYS CFX, Inc. High-performance computational fluid dynamics (CFD) software tool, http://www.ansys.com/products/fluids/ansys-cfx. 